\title{
ON A CLASS OF COMPLEX SPACES INTERMEDIATE TO STEIN AND COMPACT
}

\author{
BY ALAN T. HUCKLEBERRY ${ }^{1}$ AND RICARDO NIRENBERG \\ Communicated by Mary Ellen Rudin, March 20, 1972
}

In the global theory of complex spaces two extreme cases were traditionally studied: Compact and Stein. A pseudoconcave space represents a variation from this tradition. However, from the point of view of global function theory, pseudoconcave spaces behave much like compact spaces. For example, on a pseudoconcave space every holomorphic function is constant and the meromorphic function field is an algebraic function field having transcendence degree over $C$ bounded by the dimension of the space [1].

The $q$-pseudoconvex (concave) case, which was investigated by Andreotti and Grauert [2], represents another variation. However, the main result in the $q$-pseudoconvex case is that $\operatorname{dim}_{c} H^{r}(X, \mathscr{F})<\infty$ for $\mathscr{F}$ coherent and $r \geqq q$ [2]. Unless $X$ is Stein, nothing can be said about the lower cohomology groups or, in particular, the algebra of holomorphic functions. The main result in the $q$-pseudoconcave case is another finiteness theorem [2], but, as we have already remarked, pseudoconcave spaces behave much like compact spaces from the function theoretic standpoint.

We are therefore led to study complex spaces which at least from the global function theoretic point of view, are more clearly between the extremes of Stein and compact spaces.

Definition. Let $X$ be a complex space. We say that $X$ is $k$-pseudoflat if there is a relatively compact open subset $Y \subset X$ such that every $p \in \partial Y$ is contained in a set $S_{p} \subset \bar{Y}$, where $S_{p}$ is an analytic subvariety of an open set in $X$ such that codim ${ }_{p} S_{p} \leqq k$.

Of course the motivation for such a definition is a complex manifold containing a relatively compact open subset with smooth boundary which is Levi flat in a certain number of tangent directions.

In [4] we prove the following theorem.

THEOREM 1. Let $X$ be a reduced, irreducible $k$-pseudoflat complex space. Then there are at most $k$ analytically independent holomorphic functions on $X$.

AMS 1969 subject classifications. Primary 3222, 3247.

Key words and phrases. Pseudoflat, Levi flat, holomorphic functions, analytic independence, complex Lie groups.

${ }^{1}$ Partially supported by NSF grant GP-20139. 
As an example of an application of the theory of $k$-pseudoflat spaces we turn to complex Lie groups. Since every pseudoconcave Lie group can be shown to be a compact torus [3], it would be reasonable to expect that a complex Lie group which is neither Stein nor compact is nontrivially $k$-pseudoflat. We show in [4] that this is in fact the case.

THEOREM 2. Let $G$ be a connected, n-dimensional, complex Lie group. Suppose that $G$ is neither Stein nor compact. Then $G$ is $k$-pseudoflat for some $k$ such that $0<k<n$.

Actually a lower bound for $k$ in Theorem 2 can be determined from the dimension of the holomorphic tangent space at the identity of a maximal real torus in the level set at the identity of the functions holomorphic on $G$. For nontrivial examples of such groups we refer the reader to [5].

\section{REFERENCES}

1. A. Andreotti, Théorèmes de dépendance algébrique sur les espaces complexes pseudoconcaves, Bull. Soc. Math. France 91 (1963), 1-38. MR 27 \#2649.

2. A. Andreotti and $\mathrm{H}$. Grauert, Théorèmes de finitude pour la cohomologie des espaces complexes, Bull. Soc. Math. France 90 (1962), 193-259. MR 27 \#343.

3. A. Andreotti and A. T. Huckleberry, Pseudoconcave Lie groups, Compositio Math. (to appear).

4. A. T. Huckleberry and R. Nirenberg, On k-pseudoflat complex spaces (to appear).

5. Ahihiko Morimoto, On the classification of noncompact complex abelian Lie groups, Trans. Amer. Math. Soc. 123 (1966), 200-228. MR 34 \#706.

DePartment of Mathematics, UniVERSity of Notre Dame, NOtre Dame, INDIANa 46556

Department of Mathematics, State University of New York at Albany, Albany, NEW YORK 12203 